\title{
Lanthanide-Potassium-Biphenyl-3,3'-disulfonyl-4,4'-dicarboxylate Frameworks: Gas Sorption, Proton Conductivity, and Luminescent Sensing of Metal Ions
}

Li-Juan Zhou, ${ }^{\dagger, \S}$ Wei-Hua Deng, ${ }^{\ddagger, \S}$ Yu-Ling Wang, ${ }^{, \dagger \dagger}$ Gang Xu, ${ }^{\dagger}$ Shun-GaoYin, ${ }^{\dagger}$ and Qing-Yan Liu, ${ }^{* \dagger}$

${ }^{\dagger}$ College of Chemistry and Chemical Engineering, Key Laboratory of Small Functional Organic Molecule of Ministry of Education, , Jiangxi Normal University, Nanchang 330022, People's Republic of China

F State key laboratory of Structural Chemistry, Fujian Institute of Research on the Structure of Matter, Chinese Academy of Sciences, Fuzhou, Fujian, 350002 , People's Republic of China

${ }^{\S}$ These authors contributed equally.

\section{Supporting Information}

Table S1. Selected Bond Lengths $(\AA)$ of JXNU-2. ${ }^{a}$

\begin{tabular}{llllll}
\hline Sm & \multicolumn{3}{l}{ Eu } & & \\
\hline Sm1-O1 & $2.421(4)$ & Eu1-O1 & $2.385(4)$ & Pr1-O1 & $2.439(4)$ \\
Sm1-O3 & $2.417(4)$ & Eu1-O3 & $2.411(4)$ & Pr1-O3 & $2.467(4)$ \\
Sm1-O11 & $2.403(6)$ & Eu1-O11 & $2.380(5)$ & Pr1-O11 & $2.415(7)$ \\
Sm1-O7D & $2.421(5)$ & Eu1-O7D & $2.433(4)$ & Pr1-O7D & $2.486(4)$ \\
Sm1-O9D & $2.384(4)$ & Eu1-O9D & $2.380(4)$ & Pr1-O9D & $2.438(4)$ \\
Sm1-O1E & $2.622(4)$ & Eu1-O1E & $2.628(4)$ & Pr1-O1E & $2.669(4)$ \\
Sm1-O2E & $2.476(4)$ & Eu1-O2E & $2.464(4)$ & Pr1-O2E & $2.522(4)$ \\
Sm1-O6F & $2.523(5)$ & Eu1-O6F & $2.510(4)$ & Pr1-O6F & $2.562(4)$ \\
Sm1-O7F & $2.661(4)$ & Eu1-O7F & $2.646(4)$ & Pr1-O7F & $2.674(4)$ \\
K1-O2 & $2.564(5)$ & K1-O2 & $2.593(4)$ & K1-O2 & $2.583(5)$
\end{tabular}




$\begin{array}{llllll}\text { K1-O3A } & 2.769(5) & \mathrm{K} 1-\mathrm{O} 3 \mathrm{~A} & 2.773(4) & \mathrm{K} 1-\mathrm{O} 3 \mathrm{~A} & 2.732(5) \\ \mathrm{K} 1-\mathrm{O} 4 \mathrm{~A} & 2.687(6) & \mathrm{K} 1-\mathrm{O} 4 \mathrm{~A} & 2.658(5) & \mathrm{K} 1-\mathrm{O} 4 \mathrm{~A} & 2.701(6) \\ \mathrm{K} 1-\mathrm{O} 8 \mathrm{~B} & 2.811(5) & \mathrm{K} 1-\mathrm{O} 8 \mathrm{~B} & 2.807(5) & \mathrm{K} 1-\mathrm{O} 8 \mathrm{~B} & 2.849(5) \\ \mathrm{K} 1-\mathrm{O} 5 \mathrm{C} & 2.887(5) & \mathrm{K} 1-\mathrm{O} 5 \mathrm{C} & 2.888(5) & \mathrm{K} 1-\mathrm{O} 5 \mathrm{C} & 2.896(5) \\ & & & & \mathrm{K} 1-\mathrm{O} 1 \mathrm{~W} & 2.72(4)\end{array}$

${ }^{a}$ symmetry Codes, A: $x, y, z-1$; B: $-y+2 / 3, \quad x-y-2 / 3, \quad z-2 / 3 ; \mathrm{C}:-x+y+1, y, z-1 / 2$;

$\mathrm{D}:-y+2 / 3, x-y-2 / 3, z+1 / 3 ; \mathrm{E}:-x+y+1, y, z+1 / 2 ; \mathrm{F}: x-1 / 3, x-y-2 / 3, z-1 / 6$.

The coordinated water $(\mathrm{O} 1 \mathrm{~W})$ for $\mathbf{J X N U}-\mathbf{2}(\mathrm{Sm})$ and $(\mathrm{Eu})$ couldn't be located from the Single-crystal X-ray diffraction.

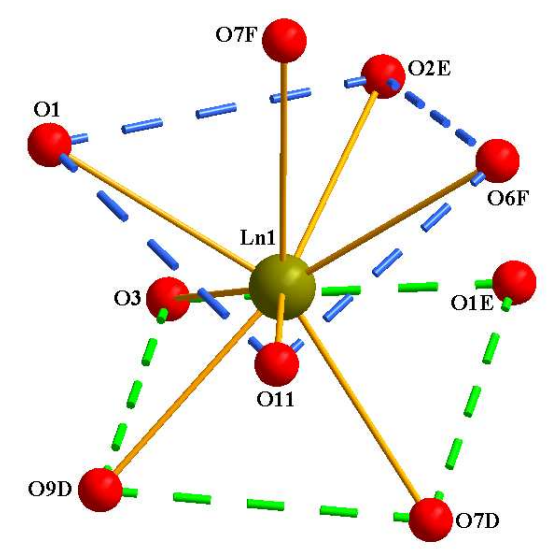

Figure S1. The coordination polyhedron of the Ln center in JXNU-2.

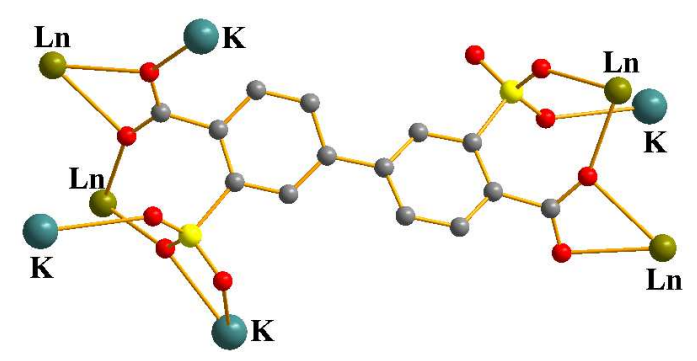

Figure S2. Coordination mode of the BPDSDC ${ }^{4-}$ ligand in compound JXNU-2. 

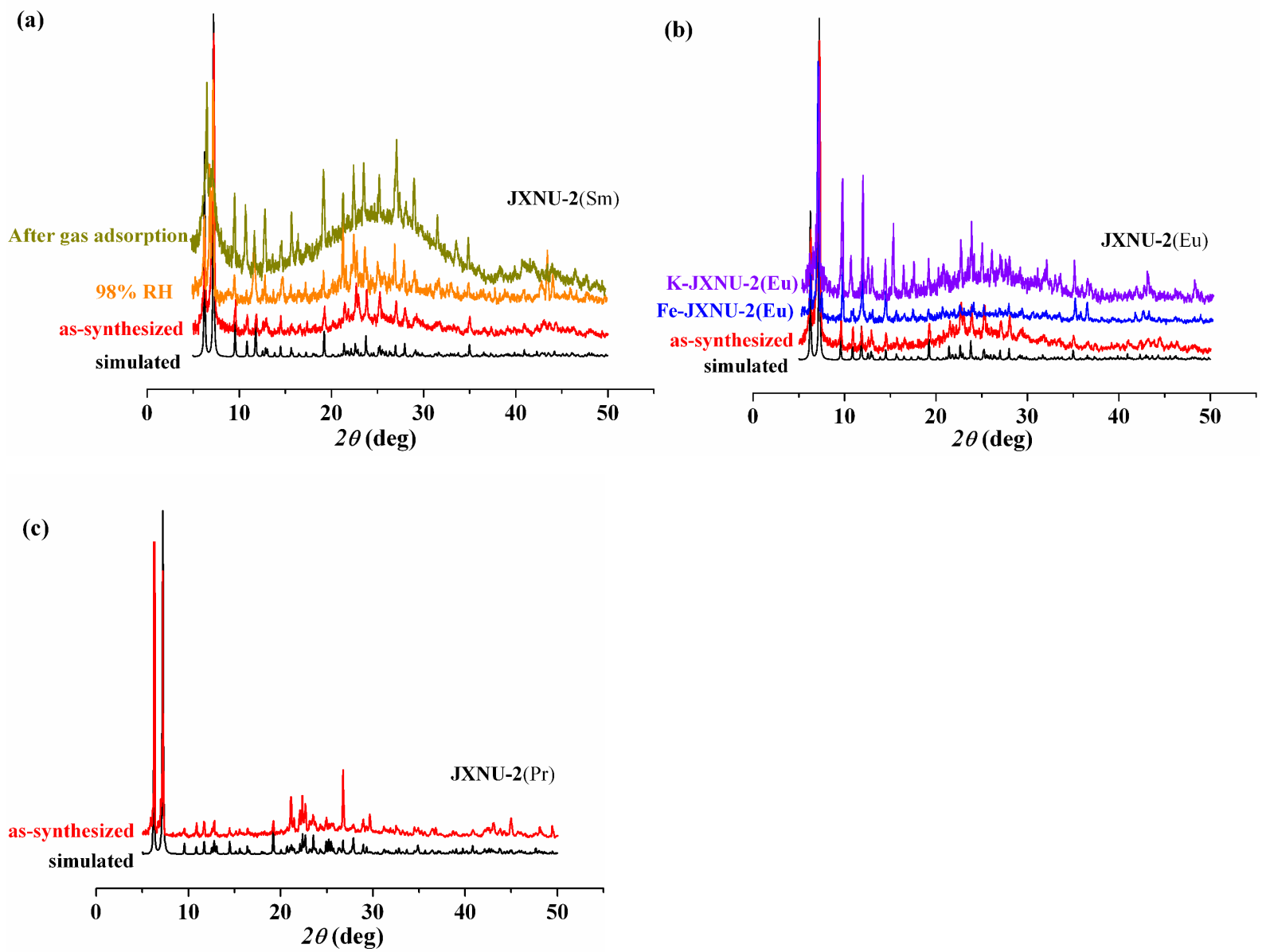

Figure S3. Simulated and experimental PXRD patterns for JXNU-2.

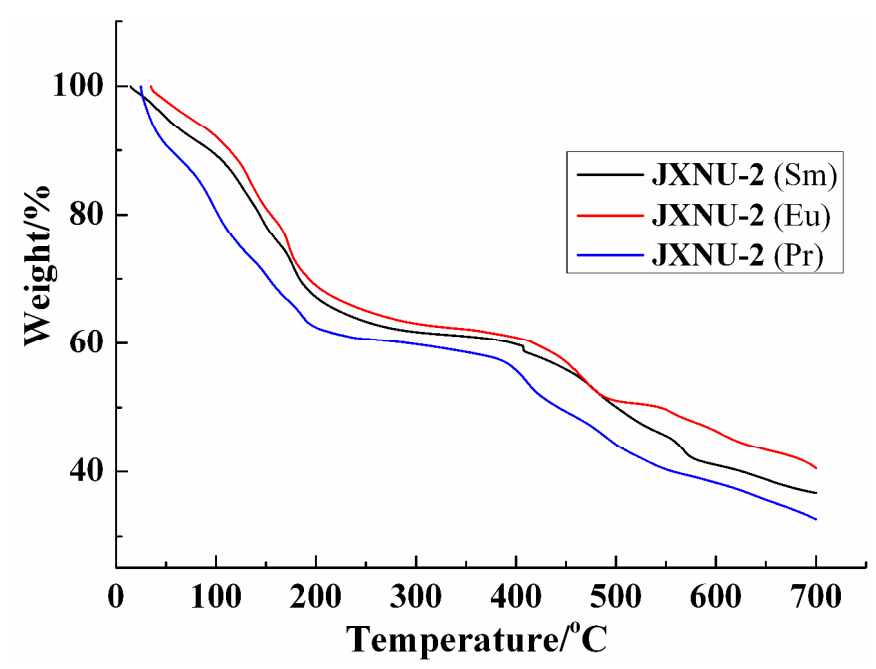

Figure S4. TG curves for JXNU-2. 


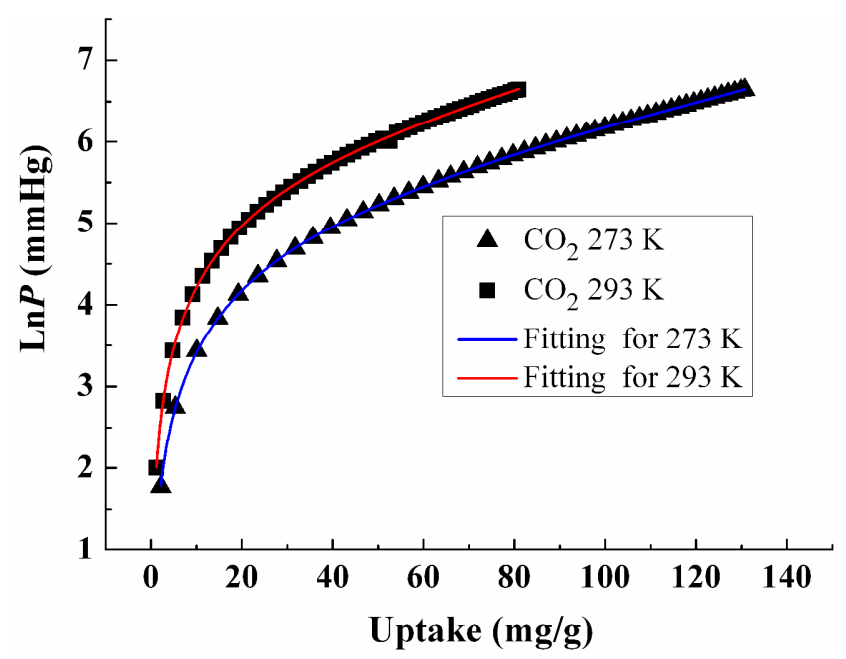

Figure S5. The Virial fit of $\mathrm{CO}_{2}$ isotherms.

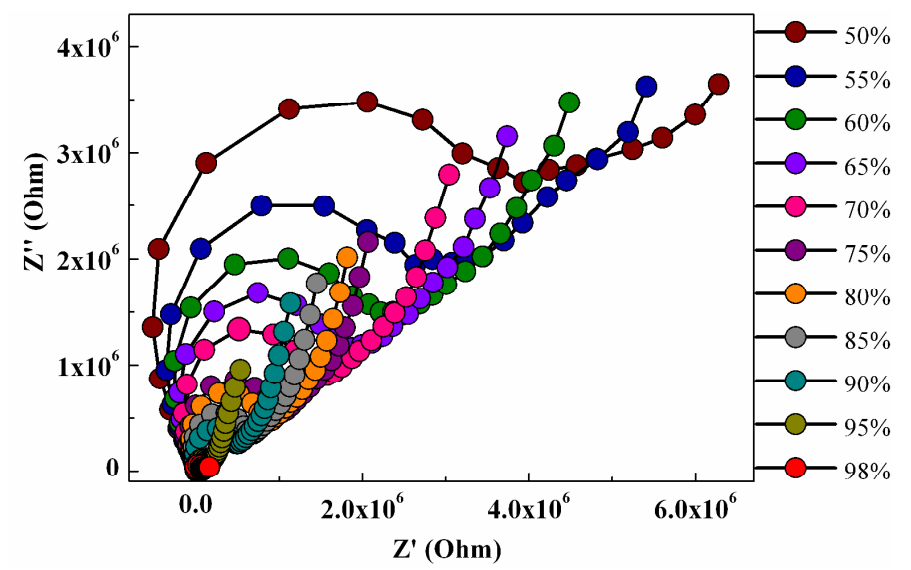

Figure S6. Nyquist plots for the pellet of JXNU-2(Sm) at $30{ }^{\circ} \mathrm{C}$ and various RH.

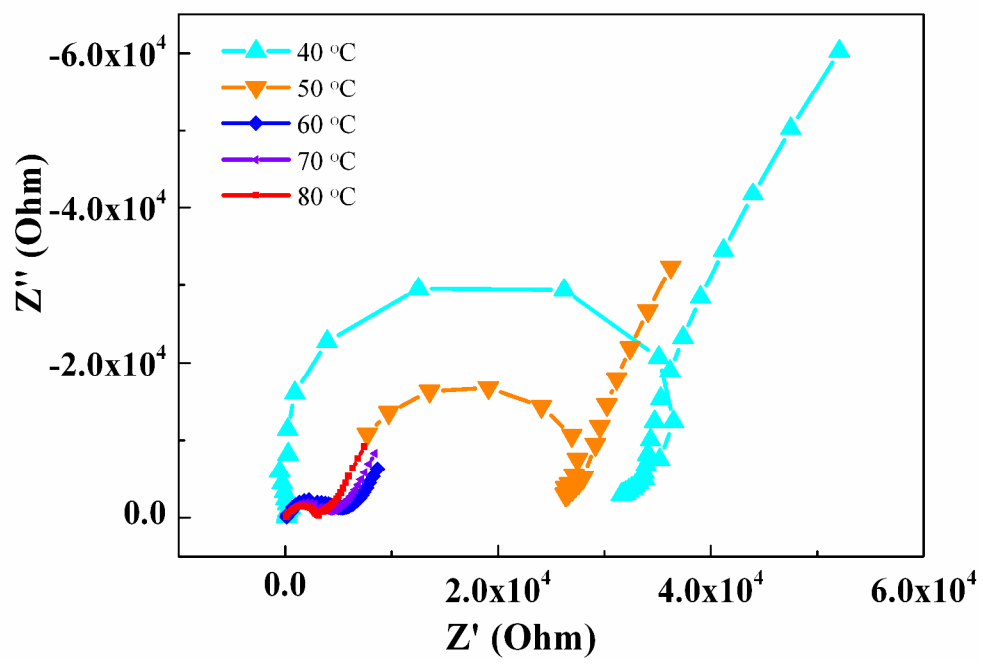

Figure S7. Nyquist plots for the pellet of JXNU-2(Sm) at 98\% RH and various temperatures. 


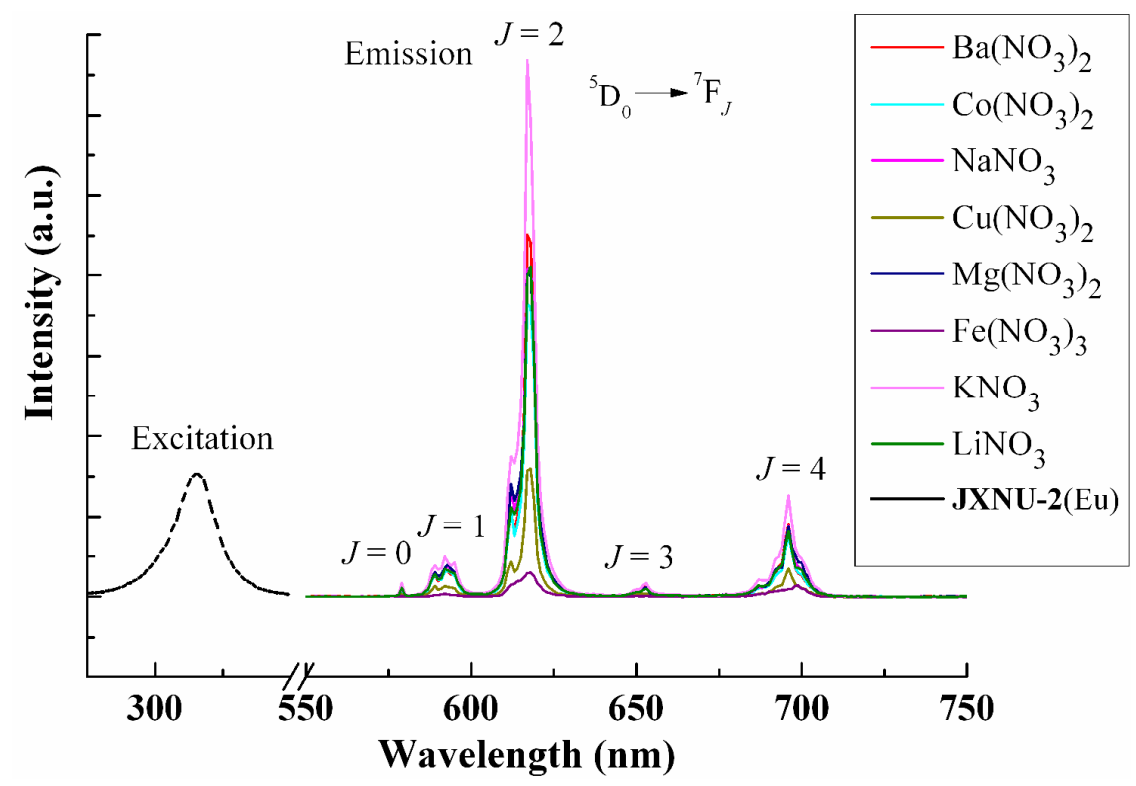

Figure S8 Excitation $\left(\lambda_{\mathrm{em}}=618 \mathrm{~nm}\right)$ and emission $\left(\lambda_{\mathrm{ex}}=316 \mathrm{~nm}\right)$ spectra of JXNU-2(Eu) and the metal-incorported JXNU-2(Eu) in DMF solutions with the metal ions concentration of $1 \mathrm{mM}$.

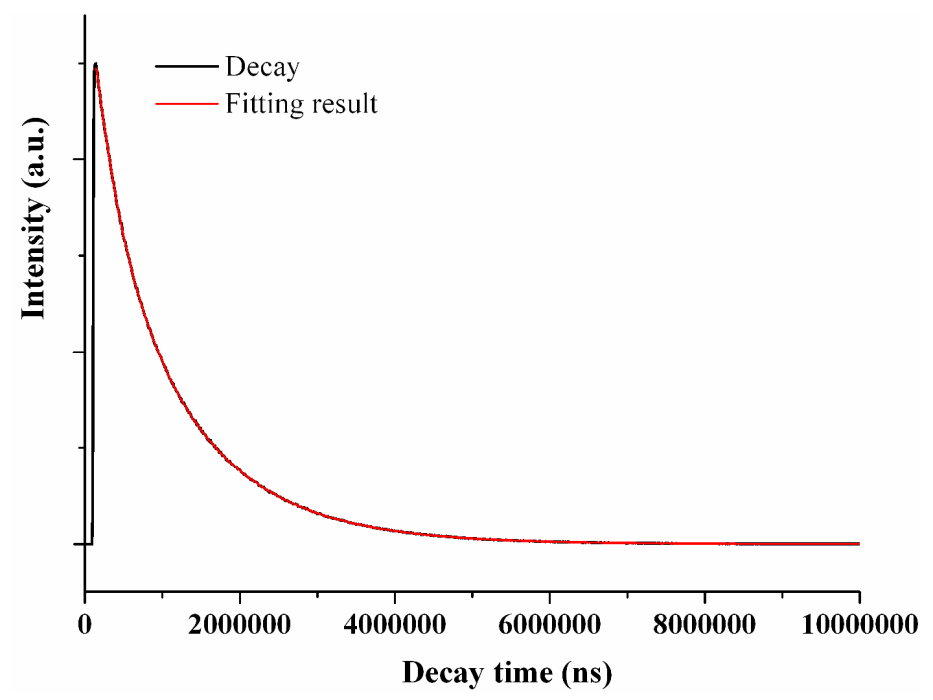

Figure S9 Luminescence decay curve of JXNU-2(Eu) in the solid state monitored within the ${ }^{5} \mathrm{D}_{0} \rightarrow{ }^{7} \mathrm{~F}_{2}$ transition. 